\title{
Efficiency of coherent vortices to trap dust particles in the solar nebula
}

\author{
Pierre-Henri Chavanis ${ }^{1 \mathrm{a}}$ \\ ${ }^{1}$ Laboratoire de Physique Théorique (IRSAMC), CNRS and UPS, Université de Toulouse, F-31062 Toulouse, \\ France
}

\begin{abstract}
We develop the idea proposed by Barge \& Sommeria (1995) that large-scale vortices present in the solar nebula can concentrate dust particles and facilitate the formation of planetesimals and planets. We introduce an exact vortex solution of the incompressible 2D Euler equation (Kida vortex) and study the motion of dust particles in that vortex. In particular, we derive an analytical expression of the capture time as a function of the friction coefficient and determine the parameters leading to an optimal capture.
\end{abstract}

\section{Introduction}

Many astrophysical objects, ranging from young stars to massive black holes, are surrounded by widespread gaseous disks. The existence of a primordial disk around the sun was conjectured by Kant and Laplace in the $18^{\text {th }}$ century to explain the quasi-circular, coplanar and prograd motion of the planets in the solar system. Such protoplanetary disks have recently been observed with the Hubble Space Telescope in the Orion nebula around stars less than one million years old.

Whenever it has been possible to observe rotating turbulent fluids with good resolution, it has been seen that individual intense vortices form. One of the most striking example is Jupiter's Great Red Spot, a huge vortex persisting for more than three centuries in the upper atmosphere of the planet. It seems therefore natural to expect their presence in accretion disks also.

Their existence was first proposed by Von Weizäcker in 1944 to explain the regularity of the planet distribution in the solar system: the famous Titius-Bode law. This idea has been reintroduced more recently by Barge and Sommeria [1] who demonstrated that anticyclonic vortices in a rotating disk are able to capture and concentrate dust particles. The capture is made possible by the action of the Coriolis force which pushes the particles inward. These results are supported by a dynamical model which integrates the motion of the particles in the velocity field produced by a full Navier-Stokes simulation of the gas component [2]. It is found that the particles are very efficiently captured and concentrated by the vortices (see Figs. 1 and 2). This is interesting because, without a trapping mechanism, $\mathrm{cm}$-sized bodies would rapidly fall onto the sun due to the inward drift associated with the velocity difference between gas and particles. Inside the vortices, the density of the dust cloud is increased by a large factor which is sufficient to trigger locally the gravitational instability and facilitate the formation of the planetesimals or the cores of giant planets. This trapping mechanism is quite rapid (a few rotations) and can reduce substantially the time scale of planet formation.

\footnotetext{
ae-mail: chavanis@irsamc.ups-tlse.fr
} 

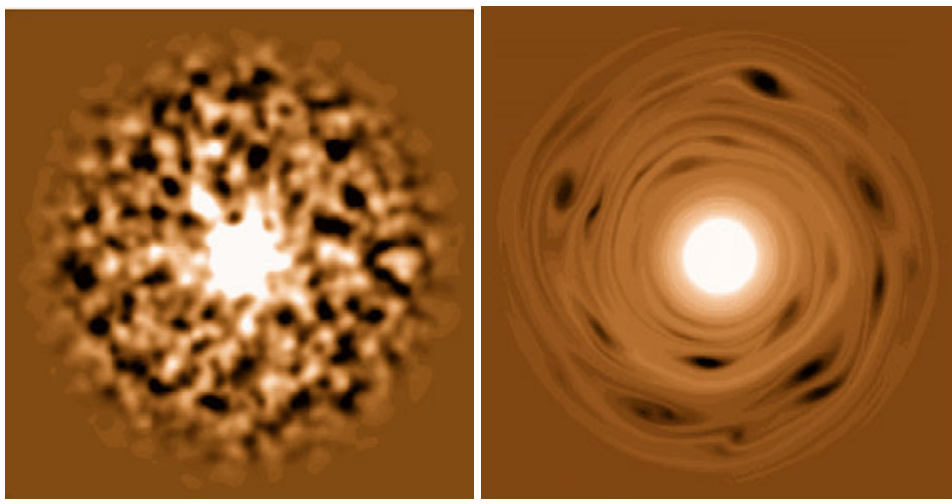

Figure 1. Spontaneous formation of anticyclonic vortices (dark spots) in a Keplerian disk surrounding a star. The disk is assumed to be initially turbulent.
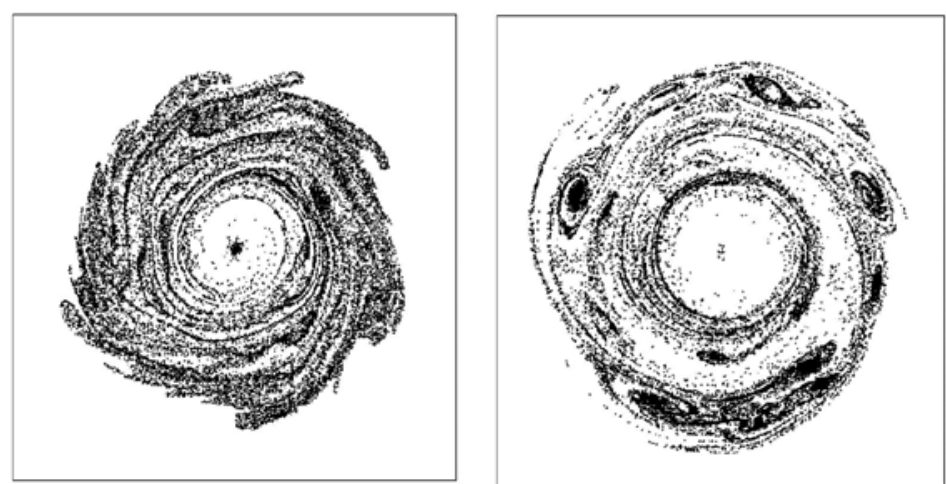

Figure 2. Concentration of dust particles inside the vortices leading ultimately to the formation of planetesimals by gravitational instability.

We present a simple analytical model for the capture of dust particles by coherent vortices in a Keplerian disk [3]. We consider an elliptic vortex with uniform vorticity matching continuously with the azimuthal Keplerian flow at large distances (Kida vortex). We study the trajectories of dust particles in that vortex and derive an analytical expression for the capture time as a function of the friction coefficient. We find that the capture is optimal for particles whose friction coefficient is close to the local disk angular velocity.

\section{The vortex model}

We consider the motion of a dust particle in a vortex located at a distance $r_{0}$ from the sun. For convenience, we work in a frame of reference rotating with constant angular velocity $\Omega \equiv \Omega\left(r_{0}\right)$ and we denote by $\mathbf{u}(\mathbf{r}, t)$ the velocity field of the gas in that frame. The dust particle is subjected to the attraction of the sun $-\frac{G M}{r^{3}} \mathbf{r}$ and to a friction with the gas that we write as $-\xi(\mathbf{v}-\mathbf{u}(\mathbf{r}, t))$ where $\mathbf{v}=\frac{d \mathbf{r}}{d t}$ is the particle velocity. Since we work in a rotating frame, apparent forces arise in the system. These are 


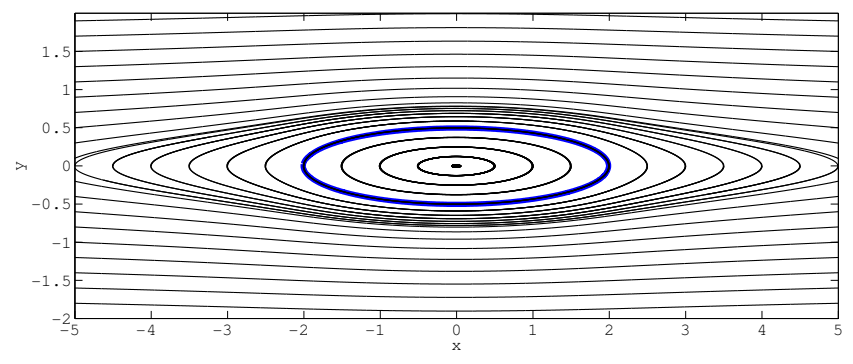

Figure 3. An example of Kida vortex with aspect ratio $q=4$ (courtesy from G. Lesur)

the Coriolis force $-2 \boldsymbol{\Omega} \times \mathbf{v}$ and the centrifugal force $\Omega^{2} \mathbf{r}$. All things considered, the particle equation reads

$$
\frac{d^{2} \mathbf{r}}{d t^{2}}=-\xi\left(\frac{d \mathbf{r}}{d t}-\mathbf{u}(\mathbf{r}, t)\right)-2 \mathbf{\Omega} \times \frac{d \mathbf{r}}{d t}+\left(\Omega^{2}-\frac{G M}{r^{3}}\right) \mathbf{r} .
$$

We introduce an exact vortex solution of the incompressible 2D Euler equation and study analytically the motion of dust particles in that vortex. Since the vortices of the solar nebula are small compared with the radial distance $r_{0}$ (we typically have $R / r_{0} \sim 0.04$ ) the last term in equation (1) can be expanded to first order in the displacement $\mathbf{r}-\mathbf{r}_{0}$. This is the so-called "epicyclic approximation". Introducing a set of cartesian coordinates $(x, y)$ centered on the vortex such that the $y$-axis points in the direction opposite to the sun, the particle equation (1) reduces to

$$
\frac{d^{2} x}{d t^{2}}=-\xi\left(\frac{d x}{d t}-u_{x}\right)+2 \Omega \frac{d y}{d t}, \quad \frac{d^{2} y}{d t^{2}}=-\xi\left(\frac{d y}{d t}-u_{y}\right)-2 \Omega \frac{d x}{d t}+3 \Omega^{2} y
$$

At sufficiently large distances from the vortex, the velocity field is a simple shear $u_{x}=\frac{3}{2} \Omega y$ and $u_{y}=0$ obtained as a first order expansion of the Keplerian velocity around $\mathbf{r}_{0}$. Its vorticity is $\omega_{K} \equiv$ $\partial_{x} u_{y}-\partial_{y} u_{x}=-\frac{3}{2} \Omega$.

It remains now to specify the velocity field. We can construct an exact vortex solution of the incompressible 2D Euler equation by considering an elliptic patch of uniform vorticity $\omega$. Outside the vortex, the vorticity is equal to the Keplerian vorticity $\omega_{K}$. This is a particular case of the so-called Kida vortex (see Fig. 3). The detailed construction of the velocity field is given in Appendix A of [3]. In the vortex, the velocity field is

$$
u_{x}=-\frac{q^{2}}{1+q^{2}} \omega y, \quad u_{y}=\frac{1}{1+q^{2}} \omega x,
$$

where $q=a / b$ is the aspect ratio of the elliptic patch ( $a, b$ are the semi-axis in the $x$ and $y$ directions respectively). Inside the vortex, the fluid particles move at constant angular velocity $q \omega /\left(1+q^{2}\right)$ along concentric ellipses with aspect ratio $q$. Outside the vortex, the velocity field is given in Appendix A of [3]. At large distances, we recover the Keplerian shear.

The matching conditions between the elliptic vortex and the Keplerian shear require that $\omega, \omega_{K}$ and $q$ be related according to (see Appendix A of [3]):

$$
\frac{\omega_{K}}{\omega}=\frac{q(q-1)}{1+q^{2}}
$$

The solution (3) is valid for $q>1$, implying $0<\omega_{K} / \omega<1$. The vortex is anticyclonic $(\omega<0)$ and is oriented with its major axis parallel to the shear streamlines. For $q \rightarrow 1$ (circular vortices), $\omega \rightarrow-\infty$ 
and for $q \rightarrow \infty$ (infinitely elongated vortices), $\omega \rightarrow \omega_{K}$. Therefore, $\omega$ is in the range $\left.]-\infty,-\frac{3}{2} \Omega\right]$. In a rotating disk, we expect that $\omega \sim-2 \Omega$, corresponding to a Rossby number of order 1 . This value is achieved by vortices with an aspect ratio $q \simeq 4$ (more precisely $q=2+\sqrt{7} \simeq 4.65$ ).

\section{The capture time}

We now derive an explicit expression for the capture time of the particles as a function of their friction coefficient $\xi$. To that purpose, we notice that equations (2) with the velocity field (3) form a linear system of coupled differential equations. We seek therefore a solution of the form $x=X e^{\lambda t}$ and $y=Y e^{\lambda t}$ where $X, Y$ and $\lambda$ are complex numbers. Substituting into equations (2) and (3), we find a fourth order polynomial equation in $\lambda$ :

$$
\lambda^{4}+2 \xi \lambda^{3}+\left(\xi^{2}+\Omega^{2}\right) \lambda^{2}+3 \frac{1+q}{q(q-1)} \xi \Omega^{2} \lambda+\frac{9}{4} \xi^{2} \Omega^{2} \frac{1}{(q-1)^{2}}=0 .
$$

By definition, we will say that a particle is light or heavy whether $\xi>\Omega$ or $\xi<\Omega$ respectively. We now consider the asymptotic limits $\xi \rightarrow \infty$ and $\xi \rightarrow 0$ of equation (5).

For $\xi \rightarrow \infty$ (light particles), the particles follow ellipses of aspect ratio $q$ and move with angular velocity $-\frac{3 \Omega}{2(q-1)}$. In fact, for $\xi \rightarrow \infty$, the drag term in equation (1) implies $\frac{d \mathbf{r}}{d t} \simeq \mathbf{u}$ so, in a first approximation, the particles just follow the vortex streamlines (their angular velocity coincides with the angular velocity of the fluid particles). In addition, they experience a drift toward the center of the vortex due to the combined effect of the friction and Coriolis forces. They reach the vortex center in a typical time

$$
t_{\text {capt }}=\frac{4 \xi}{3 \Omega^{2}} \frac{q(q-1)^{2}}{(q-2)(2 q+1)},
$$

defined as the capture time. Note that for light particles, $t_{\text {capt }}$ increases linearly with $\xi$.

For $\xi \rightarrow 0$ (heavy particles), the particles follow ellipses with aspect ratio 2 and move with angular velocity $-\Omega$. This is natural since heavy particles have the tendency to decouple from the gas and reach a pure epicyclic motion. However, due to a slight friction, they sink progressively in the vortex with a characteristic time

$$
t_{\text {capt }}=\frac{2 q(q-1)}{(q-3)(2 q+1)} \frac{1}{\xi} .
$$

For heavy particles, the capture time increases like $\xi^{-1}$. Very heavy particles can even leave the vortex.

For intermediate values of $\xi$, the capture time of the particles is plotted in Fig. 4 for the particular value $q=4$. We see that $t_{\text {capt }}$ presents a minimum at $\xi=\xi_{\text {opt }}$. Moreover, the asymptotic expressions (6) and (7) agree reasonably well with the exact solution for all the values of the friction coefficient. Therefore, considering the intersection of the asymptotes, we find that the capture time is minimum for

$$
\xi_{\text {opt }} \simeq\left[\frac{3(q-2)}{2(q-1)(q-3)}\right]^{1 / 2} \Omega, \quad t_{\text {capt }}^{\min } \simeq\left[\frac{8(q-1)^{3} q^{2}}{3(q-3)(2 q+1)^{2}(q-2)}\right]^{1 / 2} \frac{1}{\Omega} .
$$

According to equations (6) and (7), the condition for particle trapping $\left(t_{\text {capt }}>0\right)$ requires that $q>3$. This implies that the vorticity must be in the range [see equation (4)]: $-\frac{5}{2} \Omega<\omega<-\frac{3}{2} \Omega$. In particular, particles are ejected from rapidly rotating anticyclones. In the following, we shall specialize on the value of $q$ which minimizes the capture time $t_{\text {capt }}^{\min }$. We find $q \simeq 4$ (more precisely $q=5.37$ ). As noted already, this value corresponds to $\omega \simeq-2 \Omega$ (more precisely $\omega \simeq-2.125 \Omega$ for $q=4$ and $\omega \simeq-1.91 \Omega$ for $q=5.37$ ), i.e. a Rossby number of order 1 . For vortices with $q=4$ (resp. 5.37), the optimal friction coefficient is $\xi_{\text {opt }}=\Omega\left(\right.$ resp. $0.7 \Omega$ ). The corresponding capture time is $t_{\text {capt }}^{\text {min }}=\frac{8}{3 \Omega}$ 


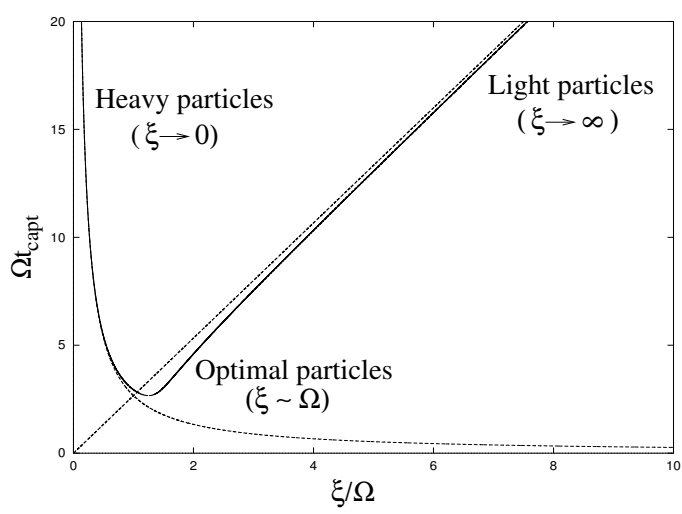

Figure 4. Capture time $t_{\text {capt }}$ as a function of the friction coefficient $\xi$ for Kida vortices with aspect ratio $q=4$. The dashed lines correspond to formulae (6) and (7) valid for light $(\xi \rightarrow \infty)$ and heavy $(\xi \rightarrow 0)$ particles.

(resp. 2.41 $\Omega^{-1}$ ); it is of the order of one rotation period. For $q=4$, we have $t_{\text {capt }} \simeq \frac{8 \xi}{3 \Omega^{2}}$ for $\xi \gg \Omega$ (light particles) and $t_{\text {capt }} \simeq \frac{8}{3 \xi}$ for $\xi \ll \Omega$ (heavy particles). Light particles move with angular velocity $-\frac{\Omega}{2}$ (the vortex velocity) and heavy particles move with angular velocity $-\Omega$ (the epicyclic velocity). Interestingly, the value of $q$ leading to an optimal capture lies in the narrow range $4<q<5.9$ where the core of the Kida vortex is stable with respect to $3 \mathrm{D}$ perturbations [4].

\section{Conclusion}

The simple model presented in this paper (see [3] for more details) shows that the capture of dust particles by coherent vortices in the solar nebula is optimal when $\xi \sim \Omega$. Using this result, one can show [3] that there exist two preferential locations in the disk where the particles are efficiently concentrated in the vortices. For the most relevant $d m$-sized particles, they turn out to coincide with the position of telluric (Earth) and giant (Jupiter) planets. The transition between the two groups of planets corresponds to the passage from the Stokes regime (inner zone) to the Epstein regime (outer zone) where the gas drag law changes. The asymmetry between the two groups of planets may be ascribed to the size of the vortices which are bigger in the outer zone and capture therefore more mass. In addition, they intercept in priority the particles drifting towards the sun.

In the last ten years, many very interesting studies related to the vortex scenario appeared. These papers have addressed the formation of the vortices, their stability and their interaction with dust particles. We refer for example to Meheut et al. [5] for a recent study and for up-to-date references.

\section{References}

[1] P. Barge, J. Sommeria A\&A 295, L1 (1995)

[2] A. Bracco, P.H. Chavanis, A. Provenzale, E.A. Spiegel, Phys. Fluids 11, 2280 (1999)

[3] P.H. Chavanis, A\&A 356, 1089 (2000)

[4] G. Lesur, J.C.B. Papaloizou, A\&A 498, 1 (2009)

[5] H. Meheut, Z. Meliani, P. Varniere, W. Benz A\&A 545, 134 (2012) 